If present plans materialize the Armour Foundation will construct one of these instruments for the University of Illinois College of Medicine, with a member of The Yerkes Observatory staff acting as consultant. This isophotometer would then be available in Chicago for use by astronomers from any institution.

I. Williams and Hiltner, Pub. Obs. Univ. Michigan 8, 45, I940.

2. von Hoff, Zs. Astroph. I4, I04, I937.

3. Garvin and Goodwin, Science I I0, 48I, 1949.

Yerkes Observatory, Williams Bay, Wis.

\section{Page, Thornton. Temperatures of the nuclei of three planetary nebulae.}

In the past, seven methods have been proposed for determining the very high temperatures of the nuclei of planetary nebulae from intensity ratios in the observable region, $\lambda \lambda_{3} 000$ to 7000 . Four of these, all due to $\mathrm{H}$. Zanstra, ${ }^{1}$ give lower limits to the nuclear temperature, $T$, based on the following intensity ratios: nebular hydrogen emission lines to nuclear spectrum, nebular $H e$ II emission $\lambda 4686$ to nuclear spectrum, forbidden emission lines of $O$ II, $O$ III, $N e$ III, $N$ II to nuclear spectrum, and difference in magnitude of nebula and star. Another method, due to V. Ambartsumian, ${ }^{2}$ is based on the ratio of nebular $H i e$ II to $H$ emission lines. It does not lead to a lower limit, and generally gives much higher values of $T$. Yet another, due to R. H. Stoy, ${ }^{3}$ is based on the ratio of nebular forbidden lines to hydrogen lines and generally yields low values of $T$. Finally, the colors of these nuclear stars in the observable region give much lower values of $T$, but would not be expected to be reliable.

Each of these methods relies on the derivation of a ratio of intensities at two wave lengths in the spectrum of the nucleus, one or both of these being calculated on the theory of fluorescence of the nebulae from the nebular emissions in the observable region. The resulting values of $T$ depend, of course, on the assumption that a Planck curve fits the nuclear spectrum; they are color temperatures derived more-or-less reliably from different parts of this spectrum.

A new method recently proposed by K. Wurm ${ }^{4}$ is based on the ratio of nebular emission in the Balmer continuum to nuclear spectrum at the same wave length, and is essentially the same as the first of Zanstra's methods, although the observational data are independent, and, because it omits line intensities, may be more accurately determined. From wide-slit spectra obtained at the $\mathrm{McD}$ Donald Observatory ${ }^{5}$ and elsewhere, this ratio has been determined for NGC 6543, NGC 6720 and NGC 7662. The resulting values of $T$ are $70,000^{\circ} \mathrm{K}, 200,000^{\circ} \mathrm{K}$ and $80,000^{\circ} \mathrm{K}$, respectively. If corrections are made for possible contamination of the nebular continuum, we obtain lower limits to $T$ of $50,000^{\circ} \mathrm{K}, \mathrm{I} 20,000^{\circ} \mathrm{K}$ and $55,000^{\circ} \mathrm{K}$, respectively. These are still considerably higher than previous determinations, except for NGC 7662 .

The comparison of all these temperature determinations leads to the conclusion, supported by other data and theory, ${ }^{4}$ that the planetary nebulae are not all of infinite optical thickness in the far ultraviolet, $\lambda<912 \mathrm{~A}$. Hence, most of the values of $T$ are much lower than the true surface temperatures of planetary nuclei. The nucleus of NGC 6720 has one of the hottest exposed surfaces yet observed.

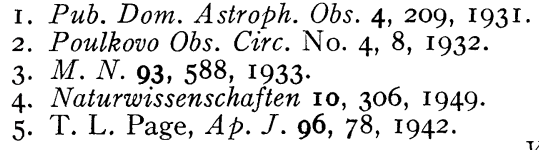
Yerkes Observatory,
Williams Bay, Wis., and McDonald Observatory, Fort Davis, Tex.

\section{Pettit, Edison. Photographing Mars.}

The polar caps of Mars and the green areas on a brick red background are easy to see, but the canals are entirely absent on most occasions. As the seeing gets very good the canals appear first singly, then a few at a time. If the moments of superseeing arrive the whole pattern will appear, but only for a second or two, at most four or five seconds. The whole interval of opportunity to see canals will cover about I5 minutes. This opportunity may be repeated several times or not at all that night, and may be had only a few times during an opposition. However, it is during these moments of superseeing that we may hope to photograph the canals with anything like the ability of the telescope to reproduce these objects. The canals are green and a yellow filter with panchromatic film should give best results. An image about $8 \mathrm{~mm}$ in diameter is desirable and for this a telescope of about 30 inches aperture is needed to get the exposure times well below the $\mathrm{I}$ - to 5 -second interval of superseeing. I think we will need an apparent image diameter in the neighborhood of $2 \mathrm{O}^{\prime \prime}$ and it is doubtful if the moments of opportunity will come except in the summer months. A stagnant atmosphere is essen- 